\title{
Metastatic Jaw Swelling as the Manifestation of Leiomyosarcoma of Uterus- A Case Report
}

\author{
MM HASAN ${ }^{1}$, M AHMED $^{2}$, RA BHUIYAN ${ }^{3}$, MM RAHMAN $^{4}$, ME MAHMUD $^{5}$
}

\section{Summary:}

Metastatic tumor in oral region is uncommon and may occur in the oral soft tissues or in the Jaw bone. Because of their rarity, metastasis in oral cavity are challenging to diagnose and treat. Oral metastasis is associated with poor prognosis. This case report is of a 45 year old female with a small pedunculated swelling on the left side of the hard

\section{Introduction:}

Metastasis to the oral cavity is uncommon and constitutes about $1 \%$ of all oral malignant tumor ${ }^{1,2,3,4}$. About $1 \%$ of the malignant tumors of the body metastasize to the oral cavity ${ }^{5}$. In the absence of any other metastasis isolated tumor seedling of oral tissues are extremely rare and constitutes $0.1 \%{ }^{6,7}$. This metastatic jaw tumor usually comes form lung, breast, genital organ, prostate, thyroid, kidney, bone and adrenals. Intra abdominal leiomyosarcoma commonly metastasize into the liver (65\%), peritoneum (21\%), lymph nodes (6\%), bone (6\%) and lung $(2 \%)^{8}$. This report describes a case of

1. Dr. Md. Mokerrom Hasan. BDS, BCS, FCPS(OMS), OSD DGHS, Deputed to Department Of Oral and Maxillofacial Surgery, Dhaka Dental College and Hospital.

2. Prof. Mohiuddin Ahmed. BDS, PhD(Japan), Head of the Department Of Oral and Maxillofacial Surgery, Dhaka Dental College and Hospital.

3. Dr. Rafique Ahmed Bhuiyan. BDS, MCPS, Dip in OMS (USSR), Assistant Professor, Department Of Oral and Maxillofacial Surgery, Dhaka Dental College and Hospital.

4. Dr. Md. Masudur Ragman. BDS, BCS, OSD DGHS, Deputed to Department Of Oral and Maxillofacial Surgery, Dhaka Dental College and Hospital.

5. Dr. Manjur-E-Mahmud. BDS, Honorary Medical Officer, Department Of Oral and Maxillofacial Surgery, Dhaka Dental College and Hospital.

Address of Correspondence: Dr. Md. Mokerrom Hasan. BDS, BCS, FCPS (OMS), Maxillofacial Surgeon, OSD DGHS, Deputed to Department of Oral and Maxillofacial Surgery, Dhaka Dental College and Hospital.

Received: 11 December, 2007

Accepted: 11 May, 2011 palate in the molar region for 30 days. Incisional biopsy revealed metastatic leiomyosarcoma with possible primaries in the uterus. Metastasis in the right lung and liver was also diagnosed. Palliative chemotherapy was started but the patient died after two weeks of diagnosis after receiving the first cycle of chemotherapy.

(J Bangladesh Coll Phys Surg 2011; 29: 96-98)

Leiomyosarcoma of uterus metastasized, to the soft tissue of upper jaw which is very rare.

\section{Case report:}

Mrs. Rahima khatun, a 45 years old woman from Pabna reported to the Oral and Maxillofacial Surgery department of Dhaka Dental College and Hospital with the complaints of a swelling on the right side of the posterior palate for 1 month with masticatory problem. The swelling was growing rapidly and was associated with pain. She had history of bleeding from the mass during mastication. Past medical history revealed the history of hysterectomy 5 months back in a private clinic of Pabna on the imaging based diagnosis of Fibroid uterus but no excisional biopsy was done. She had severe low back pain, pain on the right leg, chest heaviness but no cough or hemoptysis. She had the history of betelnut chewing, 4-5 leaves per day, for the last 10 years.

Physical examination showed an obese and anxious looking middle aged lady with moderate anemia. Her recorded blood pressure and pulse rate was 160/110 $\mathrm{mm}$ of $\mathrm{Hg}$ and 80 beats/ minute respectively. No sign of ascites or clubbing was seen but her right leg was edematous. No extra oral asymmetry was seen due to the swelling. Intra orally a soft mass was seen on the right side of the palate in the molar area which was pedunculated, pinkish in color, surface was smooth but ulcerated, non tender, measuring about $4 \mathrm{~cm} \mathrm{X} 2.5 \mathrm{~cm}$. Regional lymph node was not palpable. On examination, the chest percussion note was dull over the lower right lung and the liver was palpable which was about one 
finger breadth from the right costal margin. Orthopantomogram showed soft tissue shadow with no underlying bony erosion. ESR was $25 \mathrm{~mm}$ in first hour and liver function test showed normal enzyme level with INR of 1.25. Incisional biopsy reported spindle cells arranged in interlacing fascicles compatible with leiomyosarcoma suggesting metastasis with possible primary in uterus. Ultrasonography of whole abdomen revealed enlarged grossly heterogenous liver with multiple space occupying lesion (secondaries) with absence of uterus. Chest radiograph showed dense oval opacities in right para cardiac region and coin shadow ws seen in the right lower region of the lung with pleural effusion. X-ray lumbosacral spine showed lumbo sacral spondylitis. CT guided FNAC or liver biopsy was not performed due to poor general condition of the patient. Patient died after first cycle of chemotherapy that is two weeks after diagnosis.

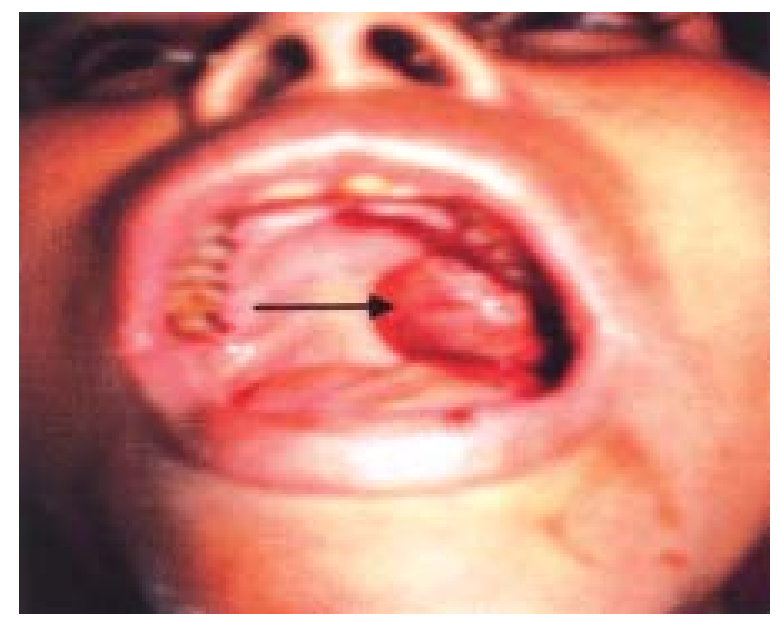

Fig-1: Intra oral photograph the swelling indicated with arrow

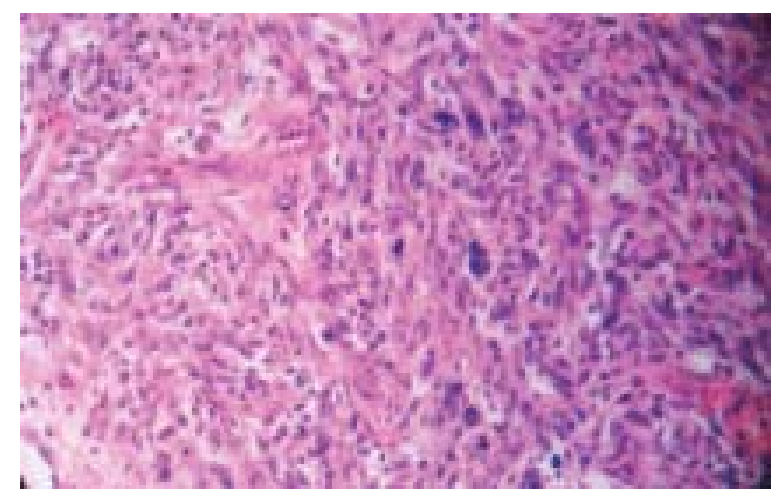

Fig.-2: Low power microscopic view of oral lesion

\section{Discussion:}

Tumors that metastasize to the oral soft tissues are very rare. The majority of the oral metastasis occur in the jaw bone (90\%) and only 5\% in the oral soft tissues ${ }^{5}$. Of the oral soft tissues 5\% occur in the tongue, $4 \%$ in the gingiva and cheek and $1 \%$ elsewhere ${ }^{5}$. Oral metastasis arises as a result of secondary spread from other metastatic lesions especially the lungs. In about $30 \%$ of oral metastasis, lung is the first site of metastatic disease. In such cases, the tumor cells bypass filtration by the lungs. Any increase in intrathoracic pressure directs blood flow into the valveless vertebral venous plexus from the azygous and caval venous system. This accounts for the increased occurrence of metastases from lung in the head neck area and axial skeleton ${ }^{9}$. Pathogenesis of metastasis to oral soft tissue is due to the rich capillary network of chronically inflammed mucosa, especially of the gingiva that can trap malignant cells. These capillaries contain fragmented basement membrane through which tumor cell can easily penetrate $^{10}$. For the most metastatic jaw tumor the primary tumor is in the breast (24\%), genital organs (17\%), lung (1296), kidney and bone $10 \%$ each $^{10}$. The report describes a case of metastatic leiomyosarcoma of oral cavity with possible primary in the uterus which is very rare. Metastasis from leiomyosarcoma to the head-and-neck, and, to the palate in particular, is unusual $^{11}$. We had to depend on the histopathology of the presented oral lesion and other history and metastatic feature for the diagnosis of primary site. CT guided FNAC and liver biopsy may have helped for diagnosis. But in this reported case it was not done due to poor general condition of the patient. Oral metastasis is considered a late complication and is commonly associated with multiple organ metastases. Oral metastasis can grow rapidly causing pain, difficulty in chewing, dysphagia, disfigurement and intermittent bleeding, leading to poor quality of life $\mathrm{e}^{1,12-16}$. Oral metastasis is an ominous prognostic sign and is associated with poor prognosis with a median survival of 4 months ${ }^{12-16}$. So treatment is aimed at palliation of symptoms. In this case the patient was given chemotherapy and the lesion was reduced in size after the first cycle of chemotherapy but the patient died after 
2 weeks of diagnosis. From the reported case it is recommended that all the oral lesions should be correlated with the thorough clinical examination and investigation of the general body condition especially when suspected for metastatic jaw swelling and any resected specimen must be sent for histopathology which may limit the subsequent disease sequele.

\section{References:}

1. Zachariades N. Neoplasms metastatic to mouth, jaws and surrounding tissues. Journal of Craniomaxill- ofacial Surgery 1989; $17: 283-290$

2. Berteli Ap, Costa FA, Miziara JFA. Metastatic tumors of the mandible. Oral Surg Oral Med Oral Pathol 1970; 30:21-24.

3. Meyer 1, Shklar G. Malignant tumors metastasis to the mouth and jaws. Oral Surg Oral Med Oral Pahol 1965; 20:350-54.

4. Stypulkowska J, Bartkowski S, Pana’s M, et al. Metastatic tumors to the jaws and oral cavity. J Oral Surg 1979;47:805809.

5. Bhaskar SN. Oral manifestation of metastatic tumors . Postgraduate Med .i 1971;49:155-157.

6. Shklar G. Oral cancer. Philadelphia, PA. Saunders. 1984.pp273-275.

7. Van der Waal RIF, Butter J, Van der Wal I. Oral metastases: report of 24 cases. Br J Oral Maxillofac Surg 2003; 41: 3-6.

8. Ronald P. DeMatteo, Jonathan J. Lewis, Denis Leuvy, et al. GIST-Recurrence patterns and Prognostic factors for survival. Ann Surgery 2000; 231: 151-58.
9. Hirshberg A, Buchner A. Metastatic neoplasms to the oral cavity. http://www.emedicine.com/derm/ topic673.htm. Accessed Jan 31,2004.

10. Hirshberg A, Leibovich P, Buchner A. Metastasis to the oral mucosa: analysis of 157 cases. Pathol Med 1993 Oct; 22(9):385-90.

11. Schenberg ME, Slootweg PJ, Koole R, et al. Leiomyosarcomas of the Oral Cavity - Report of 4 cases and review of literature. Jn Craniomaxi llofacial Surgery 1993;21C:342-7.

12. Hatziotis JC, Constantinidon H, Papanayotou PH. Metastatic tumours of oral soft tissue. Oral Surgery 1973;36:544-556.

13. Cash CD, Royer RQ, Dahlin DC. Metastatic tumours of the jaws. Oral Surgery Oral Medicine Oral Pathology Oral Radiology Endodontics 1961-114:897-905-

14. Barr CE, Dym H, Weingarten LA. Metastatic mucous producing adenocarcinoma of the gingiva. Journal of Oral Surgery 1980; 101: 53-54.

15. Kadokura M, Yamamato S, Kataoka D, et al. Pulmonary adenocarcinoma metastatic to the gingiva. Int J Clin Oncology 1999; 4: 253-255

16. Donoff RB, Albert T, Olson DJ, et al: Metastatic bronchogenic carcinoma to mandible. Jn Oral Surgery 1976; 34: 1007-1011.

17. Sanner JR, Ramin JE Yang CH. Carcinoma of the lung metastatic to gingiva-Review of literature and report of case. Oral Surgery 1979;37:103-106.

18. Hirshberg A, Buchner A.Metastatic tumours to the oral region. An overview. Eur J Cancer B Oral Oncol 1995; 31 B(6): 35560. 